\title{
Patient Reported Experience of Clinical Care of Osteogenesis Imperfecta (OI) During The COVID-19 Pandemic
}

Debra Smyth ( $\sim$ debrasmyth@hotmail.co.uk)

UofG: University of Glasgow https://orcid.org/0000-0002-4178-1435

Monica Hytiris

University of Glasgow

\section{Coreen Kelday}

The Brittle Bone Society

Ciara McDonnell

Children's Health Ireland

\section{Christine Burren}

Bristol Royal Hospital for Children

Adrian Gardner

Royal Orthopaedic Hospital NHS Foundation Trust

\section{Lisa Mills}

Bristol Royal Hospital for Children

\section{Susan Parekh}

UCL: University College London

\section{Joerg Semler}

University of Cologne: Universitat zu Koln

\section{Angie Stewart}

The Brittle Bone Society

\section{Inguun Westerheim}

Osteogenesis Imperfecta Federation Europe

\section{Kassim Javaid}

University of Oxford Nuffield Department of Orthopaedics Rheumatology and Musculoskeletal Sciences

\section{Patricia Osborne}

The Brittle Bone Society

\section{Syed Faisal Ahmed}

University of Glasgow

\section{Research Article}

Keywords: COVID-19, osteogenesis imperfecta, pandemic, rare conditions, rare diseases, remote consultation

Posted Date: December 30th, 2021

DOI: https://doi.org/10.21203/rs.3.rs-1151243/v1

License: (ㅇ) (i) This work is licensed under a Creative Commons Attribution 4.0 International License. Read Full License 


\section{Abstract}

Background Research on the effects of the COVID-19 pandemic on people with rare diseases is limited. Few studies compare healthcare throughout the progression of the ongoing pandemic.

Aims To assess the impact of lockdown on individuals with Ol (Osteogenesis Imperfecta) across two consecutive years of the pandemic, to understand what challenges were encountered, and to analyse the experience of remote consultation.

Methods Two independent surveys were distributed in August 2020 and April 2021. The primary survey was distributed following the first lockdown, and the second survey built on the experiences reported from the initial survey. The surveys explored four key themes- effects on therapy, alternatives to consultation, effect on mental health, and perceived risks of COVID-19.

Results In the primary 2020 survey of the 110 respondents 69 (63\%) had at least one appointment delayed due to lockdown, increasing to 89 of the 124 respondents ( $72 \%)$ in the follow-up survey. Of the 110 initial survey participants, $57(52 \%)$ had a remote consultation, increasing to 92 of $124(74 \%)$ in the follow-up survey. In the primary survey 63 of 91 (69\%) expressed increasing anxiety due to lockdown, compared with 76 of 124 (61\%) in the follow-up survey. In the primary survey 12 of 91 respondents (13\%) expressed concerns at not seeing friends and family compared with 103 of 124 (83\%) in the follow-up survey. In the second survey of 124 participants there were concerns regarding weight and diet $(55,44 \%)$, mobility $(70,56 \%)$, pain $(71,57 \%)$, and keeping physically active $(89,72 \%)$. The majority of participants felt they would have a worse illness and require longer rehabilitation than someone without 0 ( 81 of 117 respondents, and 77 of 120 respectively), and the majority of participants $(68 \%)$ had been vaccinated at the time of the second survey.

Conclusions The pandemic has had widespread effects on both the mental and physical health of those with OI. These repercussions are likely to be felt for years to come. On the other hand, the pandemic has also revealed that in certain situations remote consultation may prove a viable long-lasting alternative to face-to-face appointments.

\section{Background}

Although the direct effect of COVID-19 on those infected with the virus has been dramatic, the indirect effects of the pandemic on the delivery of healthcare have also been substantial and there is a need to investigate this further $(1,2)$. One group in particular who may have been affected are those with rare conditions who already felt under supported and under resourced (3) prior to this international health crisis. Rare conditions or rare diseases are defined in Europe as those conditions that affect less than 1 in 2000 people (4), with the majority being chronic, debilitating or even life threatening (4). From an organisational point of view, lockdown pressures during the recent pandemic have resulted in delays in treatment and difficulties in accessing treatment $(3,5,6)$, and additional pressures have been exerted on charities and support groups who frequently provide information and support by relying on volunteers (7). With new rules on public gatherings and social distancing, fundraising events have been cancelled resulting in financial hardships for charities (1).

Osteogenesis imperfecta (OI) is a rare collagen disorder, which has a genetic origin and often presents in childhood with skeletal fragility. It has a prevalence of about 1 in 15,000 people and can affect people to a variable extent (8). Like many rare conditions, there is no cure for Ol, however symptomatic treatments exist with the aim of optimising mobility, strengthening bones and muscles, and reducing pain and the risk of fractures $(8,9)$. Research on the effects of the COVID-19 pandemic on the health care of people with rare diseases, including OI, is limited $(1,2)$, and to improve the understanding of these effects, the Brittle Bone Society (BBS) of the UK and the Republic of Ireland, a national charity that supports individuals and families affected by Ol, performed surveys over two time periods in 2020 and 2021.

The aim of the surveys was to explore the impacts of the global COVID-19 pandemic on the osteogenesis imperfecta community, to understand the challenges they encounter throughout the ongoing pandemic, and to analyse the experience of the remote consultation on clinical care.

\section{Methods}

To measure the effects of the COVID-19 pandemic on patients with osteogenesis imperfecta, the BBS developed two online questionnaire surveys that were distributed from August to December 2020 and from March to April 2021. These questionnaires were circulated through its membership via social media (including Facebook, Twitter, Instagram), the monthly BBS newsletters and posted on its website. The second survey was also circulated via OIFE (Osteogenesis Imperfecta Federation Europe) through their membership and online. The aim of these surveys was to assess the effect of the pandemic on healthcare received by patients with Ol, but also to 
compare the effects of the pandemic between both periods. The questions were developed in conjunction with a working group of five individuals with varying types of $\mathrm{Ol}$, and with input from the BBS Medical Advisory Board and looked at the following themes: theme one - effect on therapy; theme two - alternatives to face to face consultation; theme three - effect on mental health and wellbeing; and theme four - the support from the BBS. The second survey also incorporated a novel theme; theme 5 that focused on the perceived risks of COVID-19. This was included only in the latter study due to a lack of knowledge of the effects of the COVID-19 in OI in the first wave of the pandemic when the first survey was distributed, and the unavailability of vaccines at this point in time. In the primary survey, themes were identified from answers, and these became the basis for the questions in the second survey. Some questions in the second survey were also based around the enquiries the BBS was receiving over the first year of the pandemic.

All members of the BBS (which covers the UK and Ireland) were invited to participate in the survey. Individual patients, parents and carers were invited to participate, and respondents to the questionnaire answered on behalf of the individual affected by OI. In addition, people from other countries were invited through the OIFE network. There were no exclusion criteria for participating. The questionnaire did not collect any identifiable information from respondents and was performed by the BBS. The data from respondents were supplied to the investigators on an anonymous basis for retrospective analysis. Both questionnaires contained both quantitative and qualitative aspects, with multiple choice and open-ended questions. The quantitative data were analysed using descriptive statistics, utilising Microsoft Excel, and the qualitative data were grouped using inductive thematic analysis (10).

\section{Results}

\section{Demographics}

The initial survey completed in August 2020 had 110 respondents. The follow-up survey completed in April 2021 had 124 respondents. Details of the contents of the surveys are included in Table 1. All respondents in the initial survey originated from the UK and Ireland with 85 (77\%) living in England. Of these, 80 (73\%) were individuals with OI and the remainder were a combination of parents, carers, and other relatives. The follow-up survey completed in April 2021 had 124 respondents, 96 (74\%) from the UK and Ireland, with the majority, 79 (64\%), from England. The remainder livedin Europe, South Africa, India, and the USA. Of these, 104 (84\%) respondents were individuals with Ol; 15 (12\%) were parents of a child with Ol, and 5 (4\%) were carers (Table 2).

\section{Theme 1: Effects On Therapy}

Of the 110 responses to the original 2020 survey, 69 (63\%) participants had appointments postponed due to the lock-down, and of these $19(28 \%)$ had more than one appointment postponed. Fifty-two reported a delay in a consultation, 22 had treatments deferred, and six had a surgery postponed (Fig.1.). Of the 62 respondents who gave specific feedback on how these postponements had impacted their care, the five identified themes were: delays in treatments/ investigations (21 responses, 34\%), adverse effects on health or increased pain (20 responses, $32 \%$ ), anxiety and frustration (10 responses, $16 \%$ ), and suitable arrangements were made or caused minimal effect on care (11 response, $18 \%$ ). Of the 22 paediatric patients amongst this cohort, five (23\%) had their transition to adult services impacted. Ten of the 38 respondents (26\%) requiring a wheelchair assessment were unable to have this due to the lock-down. Twenty-eight of the 54 respondents (52\%) who required rehabilitation (i.e., post surgery or following a fracture) were unable to have this, with 15 of these mentioning lack of in-person physiotherapy being their main concern. Of the 39 respondents who had educational requirements during COVID-19, 23 (59\%) felt the lock-down affected their links with educational services.

Of the 124 respondents to the follow-up 2021 survey, 89 (72\%) had appointments postponed due to the lockdown, of these 36 (40\%) had more than one appointment postponed. Twenty-two respondents (18\%) had a surgical procedure postponed or cancelled, 30 respondents $(24 \%)$ had imaging delayed/cancelled, 19 (15\%) had day unit treatment postponed/cancelled, $63(51 \%)$ had an appointment with an allied healthcare professional cancelled/postponed, and 64 (52\%) had an appointment with a specialist delayed or cancelled (Fig.2.). Twenty-five respondents had not been given a new date for their medical appointment at the time of the survey (32\%). For the postponed surgical procedures $12(48 \%)$ of respondents with delayed appointments have not been assigned a new date for their postponed procedure at the time of the survey. Of the 124 participants, $29(23 \%)$ required regular support or personal assistance at home. Four of these (14\%) had their hours of care completely cut, four ( $14 \%$ ) had the majority of their hours cut, six $(21 \%)$ had some of their hours cut, the remainder $(15,52 \%)$ experienced no change in their hours of support.

\section{Theme 2: Alternatives to consultation}

Of the 110 respondents in the 2020 survey, 57 (52\%) received remote consultations during the lockdown. For 51 (89\%) respondents this was their first experience of remote consultation. The majority of these were telephone consultations (43 responses, $75 \%$ ), eight (14\%) 
were via video consultation, and the remaining six (11\%) were a combination of both (Fig.3.). Experience with remote consultations was rated as excellent by $15(26 \%)$, very good by $13(23 \%)$, good by $17(30 \%)$, not good by $7(12 \%)$ and poor by $5(9 \%)$. When asked about future preference, 43 out of the $90(48 \%)$ respondents to this section answered they would like to have a remote consultation in the future. Of the 57 who had a remote consultation, areas for improvement that were reported included the choice of type of consultation by $9(27 \%)$, lack of examinations and blood tests by $6(18 \%)$ and not being given a time for the phone call by $4(12 \%)$. Of the 124 respondents in the 2021 survey, $92(74 \%)$ respondents had received a remote consultation/appointment. Of the 92 , telephone consultations were performed in 61 (66\%), video in 6 (7\%), and a combination in 25 (27\%) (Fig.3.). When asked about future preference, 23 respondents (19\%) said they would like to see more remote consultations, and 64 respondents (52\%) said they prefer remote consultations but not for every appointment.

\section{Theme 3: Effect on mental health and lifestyle}

In the 2020 survey, respondents were asked how COVID-19 affected them. The following themes were identified: shielding and isolation $(n=24)$; anxiety about leaving the house $(n=18)$; inability to obtain equipment/treatment $(n=12)$; reluctance to seek medical attention/ longer waits $(n=9)$; reduction in ability to exercise $(n=8)$; and financial hardship $(n=2)$. When asked specifically how the lockdown had affected their mental health, of the 91 respondents, 63 (69\%) expressed increased anxiety and depression, 12 (13\%) expressed concerns at not seeing friends and family, and two (2\%) struggled with not being physically active. Seven (8\%) respondents reported no change in their mental health due to lockdown, and six (7\%) reported a positive change in their mental health. In the 2021 survey when asked how the lockdown affected participants, of the 124 respondents, 55 (44\%) struggled moderately to significantly with weight and diet; 70 (56\%) struggled moderately to significantly with mobility; $76(61 \%)$ struggled moderately to significantly with anxiety/mental health; 103 (83\%) struggled moderately to significantly with not seeing friends and family; 71 (57\%) struggled moderately to significantly with pain; and 89 $(72 \%)$ struggled moderately to significantly with keeping physically active. For the 29 participants with carers, 22 (76\%) struggled moderately to significantly with anxiety and mental health, when compared to those who did not have carers, 54 (44\%) struggled moderately to significantly with anxiety/mental health. In the 2020 survey, of the 98 respondents, $70(63 \%)$ felt the BBS updates were very good or excellent. In the 2021 questionnaire, when asked if the BBS updates were helpful, of the 119 respondents, 69 (72\%) felt they were either extremely helpful or somewhat helpful.

\section{Theme 4: Perceived risks of COVID-19}

When asked about their general state of health in the 2021 survey, of 124 participants, 37 (30\%) felt their general health was excellent or very good, and 11 (9\%) of respondents felt their overall health was poor. Thirty-four respondents (27\%) had received an official letter from the healthcare provider advising them they were at risk of severe COVID19 related illness because of an underlying health condition, whilst 69 (56\%) self-identified as having an underlying health condition that would make them clinically vulnerable if they caught COVID19. Of the 63 who did not receive a letter, the reasons were: 25 (40\%) had not been identified as at risk (i.e.had mild OI); 13 (21\%) were unsure if they were in the at risk group but did not feel the need to register; and $9(14 \%)$ were unsure if they should be in the at risk group but did not have access to healthcare. Of the 124 respondents, 76 (61\%) had been tested for COVID-19- 22 (29\%) had severe Ol; 25 (33\%) had moderate Ol; 27 (\%) had mild disease and two (36\%) were unsure of the type of Ol they had. Of these, seven (9\%) were positive, three of these had severe disease, two had moderate disease and two had mild disease. Three respondents of $121(2 \%)$ were admitted to hospital due to having contracted COVID-19. Of the 117 who responded to the question regarding contracting COVID-19, 81 $(69 \%)$ participants felt they would be clinically more difficult to treat than someone who does not have an underlying health condition, whilst 25 (21\%) were unsure. Of the 120 who were asked about recovery from COVID-19, 77 (64\%) felt it would take them longer than someone without an underlying health condition to recover, whilst $24(20 \%)$ were unsure. When asked for further comments on recovery, 27 participants responded in total. Six respondents mentioned fear of rib fractures, from coughing or in hospital care, three mentioned that healthcare professionals have said their risk of death is high, and two mentioned fear of not being accepted into ICU or not being prioritised for care due to their disability. Due to perceived fear of COVID-19, of the 124 participants 58 (47\%) postponed their own appointments, 54 (44\%) said they had switched or changed aspects of their current therapies to avoid hospital attendance, 25 (20\%) reported that they decided not to seek out of hours emergency input at the hospital for a suspected fracture and 66 (53\%) had not left their home at all, or for most of the time since March 2020.

In the 2021 survey, of the 123 respondents to this question, $84(68 \%)$ had received the vaccine. Of the 55 participants who have a carer, 40 respondents $(73 \%)$ of their carers have been vaccinated; and of the 38 parents/family members who responded on behalf of someone with $\mathrm{Ol} 61 \%$ had been vaccinated. Of 122 respondents, $86(70 \%)$ reported that they usually get the flu vaccine and of 123 respondents, 29 (25\%) reported receiving the pneumonia vaccine. Of the 40 who had not received the COVID vaccine, 25 (63\%) had not yet been called up at the time of the survey, $4(10 \%)$ had tried to get it, but had been unsuccessful at being put on a priority list, and for

Page 4/13 
one participant from South Africa, the vaccination was not yet available in their country. When asked if respondents felt their medical needs had been considered during the vaccine rollout, of the 113 respondents to this question, $30(27 \%)$ felt like they had been ignored, $29(26 \%)$ felt they had not been given adequate consideration 21 (19\%) felt they had been given good consideration and $21(19 \%)$ felt they had been given due priority, the remainder did not feel this question was applicable to their care.

\section{Discussion}

This study explores the perception of clinical care, health and wellbeing by patients with osteogenesis imperfecta, and their carers, throughout the COVID-19 pandemic. As it stands, there are more than 262 million COVID-19 cases globally, and 5.2 million COVID-19 related deaths reported by WHO before December 1st, 2021 (11). Of these approximately one third have occurred in Europe (11). The United Kingdom currently stands as the seventh highest in reported coronavirus cases (12), where the majority (85\%) of respondents to these surveys were located.

This study has shown that delays increased from the 2020 to the 2021 survey from $63 \%$ to $72 \%$; with $28 \%$ having more than one appointment postponed increasing to $40 \%$. This could be due to the backlog of appointments across the pandemic and was associated with anxiety and frustration in one sixth of the participants. These feelings were likely to be compounded as a large proportion of those with delayed appointments were not provided with new appointment dates.

Patients with rare diseases often rely on a combination of clinical interventions including medical therapies, medications, physiotherapy, and rehabilitation services. Discontinuation of healthcare services and new lockdown measures have greatly reduced the availability of these services with an adverse effect on mental health and well-being $(1,2)$. A recent study by EURORDIS has shown that $90 \%$ of patients have experienced a delay in their clinical care since the beginning of the pandemic (5), 6 in 10 have perceived these to be detrimental, and 3 in 10 have felt these delays are life threatening. A further study showed that many patients with rare bone conditions have suffered from feelings of abandonment and fear (13).

In $89 \%$ of the initial survey participants, the lockdown resulted in their first experience of remote consultation. Only one-fifth of respondents rated the virtual consultation as 'not good or 'poor'. A recent study showed that $90 \%$ of respondents who had a teleconsultation were happy with the experience and felt it was useful (5). With over half of respondents in the first survey transitioning to remote consultation, and almost three quarters in the second wave of the pandemic, it is reassuring to see the increase in remote consultations and to know the majority of participants are still continuing treatment with a healthcare practitioner. Remote consultations existed prior to COVID-19 and had the benefit of being able to overcome geographical barriers to consultations, especially for those with rare conditions, who often have to travel to major urban areas for specialist appointments (7). However, with the arrival of COVID-19, remote consultation moved from the primary domain of the assessment of stable patients to cover those with acute and rare conditions $(14,15)$. In the initial survey approximately half of participants said that they would like more remote consultations, increasing in the follow-up survey to $71 \%$ who would prefer remote consultations either for all or some appointments. Challenges faced in remote consultations include difficulty establishing rapport, limitations to examinations and difficulty with complex concepts (7), highlighted by one fifth of participants describing challenges with lack of examinations and blood tests. Alongside these, there are also ethical and legal challenges to telehealth (1) and a requirement for patients to have digital access and expertise to join a remote consultation (16).

Those with rare conditions already experience higher levels of depression and anxiety than the general population, along with high levels of isolation and reduced interaction with family even prior to the need for shielding and social distancing $(17,18)$, Pre-existing mental health disorders have been worsened by the fear and uncertainty of the COVID-19 pandemic, and the need for shielding and social distancing has increased feelings of social isolation and loneliness $(7,19,20)$. Individuals with OI may have a reduced quality of life when compared with the general population, in particular those with more severe degree of OI reporting a worse quality of life (21). Although a pre-pandemic survey in the current cohort of participants was not available to establish a baseline, both surveys revealed that a substantial proportion of participants were struggling with anxiety. In the primary survey, when asked how COVID-19 was impacting their mental health $13 \%$ mentioned lack of contact with friends and family, increasing in the second survey to $84 \%$ when asked to rate how seeing friends and family affected them on a scale of not at all to significantly. The other theme identified in both surveys was difficulty keeping physically active. Physical therapy is also an important aspect of the management of Ol, for achieving maximal bone density but also optimising psychological wellbeing (22). Since the beginning of the pandemic, two out of three patients with rare conditions struggled with depression and/or felt unable to overcome issues (23). Previous studies investigating the effects of mental health in patients with rare conditions have shown $80 \%$ of patients have suffered during the pandemic, with those who are dependent affected more than those who live life independently (2). This is echoed in our study, which showed that the percentage of those struggling with mental health increased from $61 \%$ to $76 \%$ for those with carers. Almost half of the respondents who required regular

Page 5/13 
support or personal assistance at home had their hours either completely or partially reduced, which can create challenges at home with significant stress for the primary carers (24).

Whilst the UK Government did not specifically mention OI as a clinically extremely vulnerable group that should shield (25) some people with OI may be considered to be at a greater risk of more severe disease and pulmonary complications (26). Almost one third of participants in this study received a public health letter advising they were at risk of severe illness secondary to COVID-19, but over half had to self-identify as being at risk, due to underlying health conditions. In other research, $60 \%$ of people with rare conditions stated they had difficulty obtaining the information they required on COVID-19 (23). A substantial proportion of people with OI changed their behaviour around seeking medical help with a fifth not attending hospital for a suspected fracture and almost half changing their therapies or appointments to avoid attending hospital. This could have ledto greater pain and discomfort due to a lack of appropriate immobilisation and may have increased the potential for a poorer outcome long term. Additionally, over half of respondents to the first survey had rarely left their home since the start of the pandemic in March 2020.

By the time of the April 2021 survey, the majority of participants had received their first vaccine. However, over half of respondents felt that their medical needs were not prioritised correctly with regards to vaccination rollout. This is not an uncommon experience for those with rare conditions, as knowledge of their conditions is often limited (27). During the pandemic, the BBS provided online COVID-19 information to its members, with the majority of respondents finding this very helpful. Previous studies have shown that online support from patient organisations is welcomed amongst patients with rare diseases $(3,28)$ and in the case of some rare conditions are potentially the only source of information (29). With patient organisations being described as the major lifeline for many of the rare disease communities (1), it is unfortunate that many of these are currently working at reduced or no capacity due to decreased staffing, lack of fundraising, and diminished government subsidies (7).

Although one of the strengths of this study was the comparison of opinions across two consecutive years of the pandemic, unfortunately, we do not have a pre-pandemic survey in this cohort of participants to establish a baseline, which is particularly important when trying to assess differences in mental health and therapy options. The survey's questions also changed significantly from year to year, making direct comparison challenging. As with many qualitative studies, however, there are numerous instances of missing data (1). Furthermore, this study did not look at the delay in diagnosis as the survey was only circulated to those with known Ol. It would also be helpful to explore the impact of the pandemic on carers. Lastly, the majority of responses related to adults with Ol, with only 11 respondents within the childhood age bracket and adequate comparison to children would have required a larger number of cases.

In summary, these surveys have revealed that the pandemic has had widespread effects on both the mental and physical health of those with OI. However, the emergence of remote consultation as a direct consequence of COVID-19 may prove a viable long-lasting alternative to in-person appointments, especially for patients with OI patients who have previously travelled vast distances for specialist care. It would be beneficial to perform this survey at occasional but regular intervals to assess the on-going impact of COVID-19, and to explore pandemic related healthcare in a wider range of countries, to obtain a broader and more global perspective.

\title{
Abbreviations
}

\author{
A\&E - Accident and Emergency \\ AHP - Allied Health Practitioner \\ BBS - Brittle Bone Society \\ EURORDIS - European Rare Diseases Organisation \\ GP - General Practitioner \\ ICU - Intensive Care Unit \\ MRI - magnetic resonance imaging \\ OI - Osteogenesis Imperfecta \\ OIFE - Osteogenesis Imperfecta Federation Europe \\ RD - Rare Disease
}


UK - United Kingdom

WHO - World Health Organisation

\section{Declarations}

Ethics Approval and Consent to Participate - All responses to the surveys were anonymous. The Brittle Bone Society has a Data Protection Policy, adheres to GDPR (General Data Protection Regulation)regulations, and is registered with the ICO (Information Commissioner's Office).

Consent for Publication - Not applicable.

Availability of Data and Materials - The data was made available from the BBS.

Competing Interests - None

Funding -BBS were awarded sponsorship from Alexion Pharmaceutical to carry out the surveys.

Authors Contribution - MH, DS, CK and SFA undertook the initial data analysis and wrote the initial draft. The BBS developed both questionnaires and distributed these to participants. All authors contributed to the revision of the manuscript and have read and approved the final report. All authors take public responsibility and accountability for the results.

Acknowledgements - We would like to acknowledge the participants for taking their time to complete the surveys.

\section{References}

1. Chung C, Ng Y, Jain R, Chung B. A thematic study: impact of COVID-19 pandemic on rare disease organisations and patients across ten jurisdictions in the Asia Pacific region. Orphanet Journal of Rare Diseases.

2021;16(1). https://ojrd.biomedcentral.com/articles/10.1186/s13023-021-01766-9

2. Chung C, Wong W, Fung J, Hong Kong R, Chung B. Impact of COVID-19 pandemic on patients with rare disease in Hong Kong. European Journal of Medical Genetics.

2020;63(12):104062. https://www.sciencedirect.com/science/article/abs/pii/S1769721220307722?via\%3Dihub

3. Baumbusch J, Mayer S, Sloan-Yip I. Alone in a Crowd? Parents of Children with Rare Diseases' Experiences of Navigating the Healthcare System. Journal of Genetic Counselling. 2018;28(1):80-90. https://onlinelibrary.wiley.com/doi/full/10.1007/s10897-0180294-9

4. Eurordis Rare Diseases Europe. About rare diseases. https://www.eurordis.org/about-rare-diseases. Accessed 5th July 2021.

5.9 in 10 people living with a rare disease experiencing an interruption in care because of COVID-19 [Internet]. EURORDIS Rare Diseases Europe; 2021 [cited 6 July 2021]. Available from:

https://download2.eurordis.org/documents/pdf/PressRelease_COVID19surveyresults.pdf

6. Hytiris M, Johnston D, Mullen S, Smyth A, Dougan E, Rodie M et al. Experience of health care at a reference centre as reported by patients and parents of children with rare conditions. Orphanet Journal of Rare Diseases. 2021;16(1).

7. Making the Unseen Seen, Rare disease and the lessons learned from the COVID-19 pandemic [Internet]. Action for Rare Disease Empowerment; 2021 [cited 6 July 2021]. Available from: https://www.camraredisease.org/wp-content/uploads/2021/05/ARDEntReport-Making-the-Unseen-Seen-May-2021-FINAL.pdf

8. European Organisation for Rare Diseases. The voice of 12,000 patients: experiences and expectations of rare disease patients on diagnosis and care in Europe; 2009. https://www.eurordis.org/IMG/pdf/voice_12000_patients/EURORDISCARE_FULLBOOKr.pdf. Accessed 2 June 2020.

9. Osteogenesis Imperfecta - NORD (National Organization for Rare Disorders) [Internet]. NORD (National Organization for Rare Disorders). 2007 [cited 6 July 2021]. Available from: https://rarediseases.org/rare-diseases/osteogenesis-

imperfecta/\#: :text=In\%20most\%20cases\%2C\%20osteogenesis\%20imperfecta,this\%20mutation\%20is\%20autosomal\%20dominant.

10. Javadi M, Zarea K. Understanding thematic analysis and its pitfall. Demo. 2016 Mar 25;1(1):33-9.

11. World Health Statistics 2021: A visual summary [Internet]. World Health Organisation. 2021 [cited 6 July 2021]. Available from: https://www.who.int/data/stories/world-health-statistics-2021-a-visual-summary

Page $7 / 13$ 
12. Countries where Coronavirus has spread - Worldometer [Internet]. Worldometers.info. 2021 [cited 6 July 2021]. Available from: https://www.worldometers.info/coronavirus/countries-where-coronavirus-has-spread/

13. Brizola E, Adami G, Baroncelli G, Bedeschi M, Berardi P, Boero S et al. Providing high-quality care remotely to patients with rare bone diseases during COVID-19 pandemic. Orphanet Journal of Rare Diseases. 2020;15(1). https://ojrd.biomedcentral.com/articles/10.1186/s13023-020-01513-6

14. Wherton J, Shaw S, Papoutsi C, et al. Guidance on the introduction and use of video consultations during COVID-19: important lessons from qualitative research. BMJ Leader 2020;4:120-123. https://bmjleader.bmj.com/content/4/3/120? $\mathrm{fbclid=IwAROIqzesTzya1vXfxuMMw0z6M8IQmSp23Es1FjOzDliXmjsa2-XztcWjGH8 \# ref-8}$

15. Greenhalgh T, Wherton J, Shaw S, Morrison C. Video consultations for covid-19. BMJ. 2020;m998. https://www.bmj.com/content/368/bmj.m998? utm_term=tbmj\&utm_content=BMJUK_TMD_CM_2021\&utm_campaign=usage\&utm_medium=trendmd\&utm_source=trendmd

16. Henderson A. Indirect effects of the pandemic on UK health I LSHTM [Internet]. London School of Hygiene and Tropical Medicine. 2021 [cited 6 July 2021]. Available from: https://www.Ishtm.ac.uk/newsevents/expert-opinion/indirect-effects-pandemic-uk-health

17. Global Genes. Rare disease impact report: insights from patients and the medical community; 2013. https://globalgenes.org/wpcontent/uploads/2013/04/ShireReport-1.pdf. Accessed 4 July 2021

18. Nunn R. "It's not all in my head!" - The complex relationship between rare diseases and mental health problems. Orphanet Journal of Rare Diseases. 2017;12(1). https://ojrd.biomedcentral.com/articles/10.1186/s13023-017-0591-7

19. Kontoangelos K, Economou M, Papageorgiou C. Mental Health Effects of COVID-19 Pandemia: A Review of Clinical and Psychological Traits. Psychiatry Investigation. 2020;17(6):491-505. https://www.ncbi.nlm.nih.gov/pmc/articles/PMC7324731/

20. Campion J, Javed A, Sartorius N, Marmot M. Addressing the public mental health challenge of COVID-19. The Lancet Psychiatry. 2020;7(8):657-659. https://www.thelancet.com/journals/lanplh/article/PIIS2215-0366(20)30240-6/fulltext

21. 21 Dahan-Oliel N, Oliel S, Tsimicalis A, Montpetit K, Rauch F, Dogba M. Quality of life in osteogenesis imperfecta: A mixed-methods systematic review. American Journal of Medical Genetics Part A. 2015;170(1):62-76.

22. Exercise and Activity: Key Elements in the Management of OI [Internet]. National Institutes of Health | Osteoporosis and Related Bone Diseases National Resource Center. 2015 [cited 6 July 2021]. Available from: https://www.bones.nih.gov/healthinfo/bone/bone-health/exercise/exercise-and-activity-key-elements-management-oi

23. How has COVID-19 impacted people with rare diseases? [Internet]. 2020 [cited 6 July 2021]. Available from: https://download2.eurordis.org/rbv/covid19survey/covid_infographics_final.pdf

24. Lafferty A, Phillips D, Dowling-Hetherington L, Fahy M, Moloney B, Duffy C et al. Colliding worlds: Family carers' experiences of balancing work and care in Ireland during the COVID-19 pandemic. Health \& Social Care in the Community. 2021. https://onlinelibrary.wiley.com/doi/full/10.1111/hsc.13365

25. Guidance on shielding and protecting people who are clinically extremely vulnerable from COVID-19 [Internet]. GOV.UK. 2021 [cited 6 July 2021]. Available from:https://www.gov.uk/government/publications/guidance-on-shielding-and-protecting-extremelyvulnerable-persons-from-covid-19/guidance-on-shielding-and-protecting-extremely-vulnerable-persons-from-covid-19\#cev

26. Wagner S. COVID-19 - info for people with OI [Internet]. OIFE - Osteogenesis Imperfecta Federation Europe. 2020 [cited 6 July 2021 ]. Available from:https://oife.org/2020/12/09/corona-info-for-people-with-oi/

27. Von der Lippe C, Diesen P, Feragen K. Living with a rare disorder: a systematic review of the qualitative literature. Molecular Genetics \& Genomic Medicine. 2017;5(6):758-773. https://onlinelibrary.wiley.com/doi/full/10.1002/mgg3.315

28. Nicholas D, Chahauver A, Brownstone D, Hetherington R, McNeill T, Bouffet E. Evaluation of an Online Peer Support Network for Fathers of a Child With a Brain Tumor. Social Work in Health Care. 2012;51(3):232-

245. https://www.tandfonline.com/doi/abs/10.1080/00981389.2011.631696

29. Rare Disease UK. What is a rare disease? 2018. https://www.raredisease.org.uk. Accessed 3 July 2021.

\section{Tables}

Table 1- Survey Questions

Contents of 2020 and April 2021 surveys including responses available 


\section{August 2020 Survey Questions}

\section{Theme 1: Effects on Therapy}

Have you had any Ol appointments postponed due to COVID-19?

If yes, what was it for?

\section{April 2021 Survey Questions}

Have you/the individual with Ol ever had a surgical procedure postponed or cancelled due to COVID-19 pandemic by the Hospital?

If you have had a surgical procedures postponed on average how long have these been postponed by in months?

How has this impacted you?

I/the individual with OI have had a scan, such as an x-ray, DXA, MRI delayed or cancelled due to the COVID-19 pandemic by the Hospital

Has COVID-19 impacted your transition to adult services?

I/the individual with OI have had a day unit treatment, such as IV infusion delayed or cancelled due to the COVID-19 pandemic by the Hospital

Has COVID-19 impacted your ability to get a wheelchair assessment?

I/the individual with OI have had an appointment with an Allied Healthcare professional (e.g.: physio, OT, nurse, dental, hearing test) delayed or cancelled due to the COVID-19 pandemic by the hospital

Has COVID-19 impacted your rehab? i.e. post surgery/fracture.

I/the individual with OI have had an appointment with a Consultant/Specialist delayed or cancelled due to the COVID-19 pandemic by the Hospital

Has COVID-19 impacted how you liaise with schools/further education?

If you have had any appointment postponed on average how long have these postponed by in months?

If you receive support/care or personal assistance at home on a regular basis to what extent has this been impacted by the pandemic?

\section{Theme 2: Alternatives to consultation}

Have you received Remote Consultations during lockdown?

Was the remote consultation/appointment via:

If Yes, was this your first remote consultation?
Have you/the individual with Ol received remote consultations/appointments during the COVID-19 pandemic?

Was the remote consultation/appointment via:

Going forward is this something you would like to see more of?

I/the individual with OI prefer a hospital appointment via telephone/video call to a face to face visit

If Yes, how did you find this?

Going forward, is this something you would

like to see more of?

Would you like to suggest any ways this experience could have been improved or do you have further comments?

\section{Theme 3: Effect on mental health}

How has COVID-19 affected you?

Please indicate on a scale of 1 to 5 how much you have struggled/or not struggled with the following

$>$ Weight and diet

$>$ Mobility

$>$ Pain

$>$ Anxiety/mental health

$>$ Keeping physically active

$>$ Not seeing friends and family

Please explain the impact COVID-19 has had

on your mental health 
Theme 4: Perceived risk of COVID-19 (only addressed in April 2021 Survey)

In general would you say you/the person with Ol's health is:

Have you/ the individual with Ol received a letter from the NHS saying you have been identified as someone at risk of severe illness if you catch COVID 19 because of underlying health condition?

If you answered no to this is it because:

Have you/the individual been tested for COVID-19?

Do you have any underlying health condition that would also identify you as being clinically extremely vulnerable if you caught COVID-19?

Have you/ the individual been admitted to hospital due to having COVID-19?

In the event that you have either have had COVID-19 or may catch COVID-19, in your opinion do you think you would be clinically more difficult to treat than someone that does not have any underlying health condition?

In the event that you have either had COVID-19 or may catch COVID-19 - in your opinion do you think it did/would take you:

Have you/the individual with OI ever cancelled or postponed an appointment or treatment due to COVID-19?

Have you/the individual with $\mathrm{Ol}$ ever had to switch or change any aspect of your current therapies to avoid hospital attendance due to COVID-19?

Have you/the individual with Ol ever decided not to go to Accident and Emergency with a suspected fracture to confirm diagnosis during the COVID-19 pandemic?

To what extent has the pandemic affected you/the families ability to leave the house

Have you/ the individual with OI had a COVID-19 vaccine?

If you/the individual with OI has not had the vaccine why is this?

Table 2 - Patient Demographics 


\begin{tabular}{|c|c|c|c|}
\hline 2020 Survey $(n=110)$ & $\mathbf{N}$ & 2021 Survey $(n=124)$ & $\mathbf{N}$ \\
\hline \multicolumn{4}{|l|}{ Individual/parent/carer of someone with OI } \\
\hline Individual with OI & 80 & Individual with OI & 104 \\
\hline Parent of someone with OI & 15 & Parent of someone with Ol & 15 \\
\hline Parent of someone with $\mathrm{OI}$ and individual with $\mathrm{OI}$ & 12 & Carer of someone with OI & 5 \\
\hline Parent and carer of someone with $\mathrm{Ol}$ & 2 & & \\
\hline Other & 1 & & \\
\hline \multicolumn{4}{|l|}{ Country of residence } \\
\hline England & 85 & England & 79 \\
\hline Scotland & 11 & Other & $28^{*}$ \\
\hline Northern Ireland & 6 & Scotland & 10 \\
\hline Wales & 4 & Ireland & 4 \\
\hline Ireland & 2 & Northern Ireland & 2 \\
\hline No response & 2 & Wales & 1 \\
\hline \multicolumn{4}{|l|}{ Severity of OI } \\
\hline \multirow[t]{4}{*}{ Not asked in this survey } & & Mild & 36 \\
\hline & & Moderate & 47 \\
\hline & & Severe & 36 \\
\hline & & Unsure & 5 \\
\hline \multicolumn{4}{|l|}{ Age of individual } \\
\hline \multirow[t]{5}{*}{ Not asked in this survey } & & Ages $<15$ & 11 \\
\hline & & Ages 18-30 & 12 \\
\hline & & Ages $31-50$ & 54 \\
\hline & & Ages $50-64$ & 39 \\
\hline & & >Ages 64 & 8 \\
\hline
\end{tabular}

* Including Norway, Sweden, Denmark, Netherlands, Malta, Finland, India, USA, Belgium, South Africa

\section{Figures}


Delays in Treatment - 2020 Survey

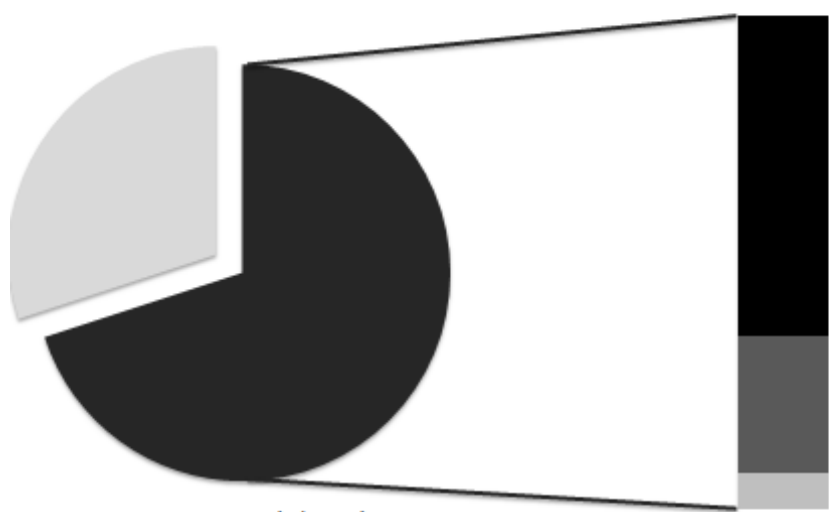

- Appointment delayed

Appointment not delayed
- Consultation

- Treatment

w Surgery

\section{Figure 1}

Delays in Treatment 2020 Survey. In the 2020 primary survey, 69 participants (63\%) hadappointments postponed due to lockdown, with $19(28 \%)$ having more than one consultation delayed. There were 80 delayed consultations in total, of these $52(65 \%)$ were a delay in consultation, $22(27.5 \%)$ had treatment delays and six (7.5\%) were surgical delays.

\section{Delays in Treatment-2021 Survey}

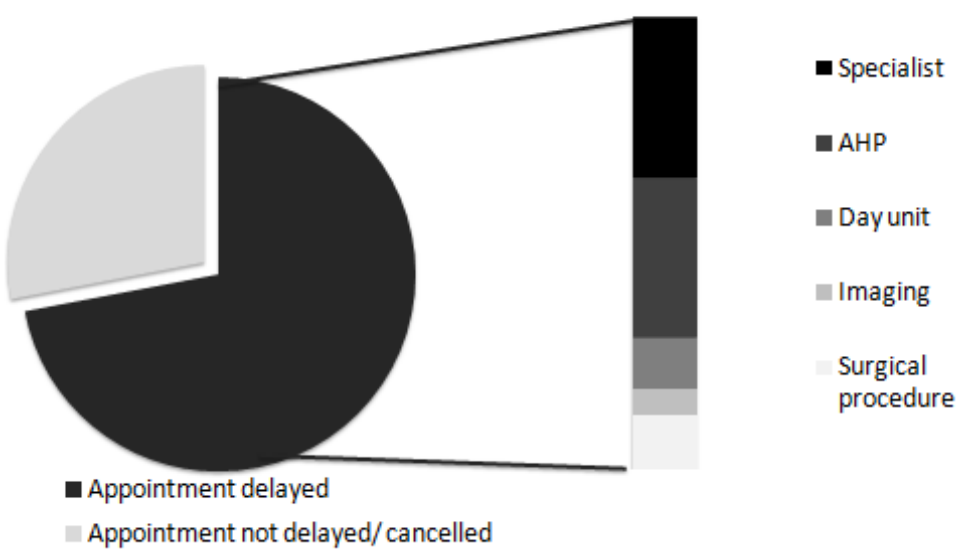

Figure 2

Delays in Treatment 2021 Survey. In the 2021 survey, 89 participants (72\%) had appointments postponed or cancelled due to COVID-19 lockdown with $36(40 \%)$ having more than one appointment delayed. There were 98 delayed/cancelled appointments in total, of these 64 (32\%) were delayed/cancelled appointments with a specialist, 63 (32\%) were delayed/cancelled AHP appointments, 19 (10\%) were delayed/cancelled day unit treatments, 30 (15\%) were delayed/cancelled imaging, and 22 (11\%) were delayed/cancelled surgical procedures. 


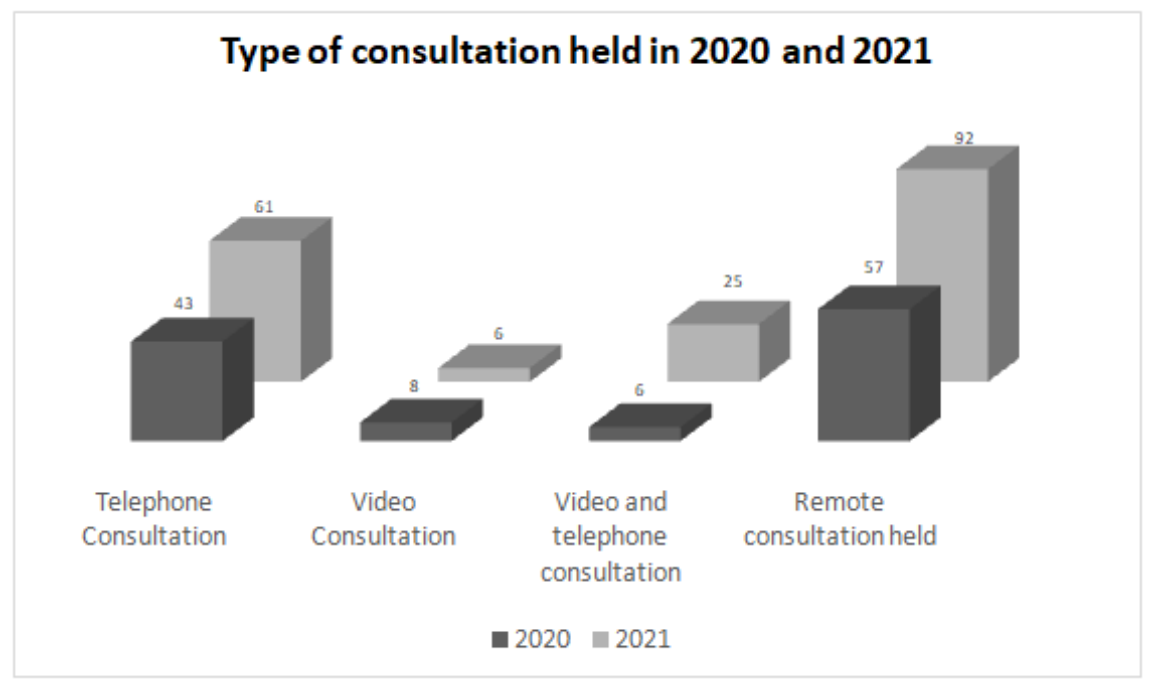

\section{Figure 3}

Type of consultation from 2020 to 2021. Comparison of consultation types from 2020 to 2021 survey. Overall, there were 57 remote consultations amongst 110 respondents 2020 and 92 amongst 124 respondents in 2021. In the primary survey, 43 respondents had telephone consultations, compared with 61 in the 2021 survey. Eight respondents had a video consultation in 2020 and a further six in 2021. Six participants had a combination of video and telephone consultations in 2020 and a further 25 in the 2021 survey. 